\title{
LSTM Sinir Ağı ve ARIMA Zaman Serisi Modelleri Kullanılarak Bitcoin Fiyatının Tahminlenmesi ve Yöntemlerin Karşılaştırılması
}

\author{
Sezercan Tanışman ${ }^{1 *}$, Abdullah Ammar Karcıoğlu², Aybars Uğur ${ }^{3}$, Hasan Bulut ${ }^{4}$ \\ ${ }^{1 *}$ Ege Üniversitesi, Mühendislik Fakültesi, Bilgisayar Mühendisliği Bölümü, İzmir, Türkiye, (ORCID: 0000-0002-8094-708X), sezercan.tanisman@ege.edu.tr \\ 2 Ege Üniversitesi, Mühendislik Fakültesi, Bilgisayar Mühendisliği Bölümü, İzmir, Türkiye (ORCID: 0000-0002-0907-751X), abdullah.ammar.karcioglu@ege.edu.tr \\ ${ }^{3}$ Ege Üniversitesi, Mühendislik Fakültesi, Bilgisayar Mühendisliği Bölümü, İzmir, Türkiye (ORCID: 0000-0003-3622-7672), aybars.ugur@ege.edu.tr \\ ${ }^{4}$ Ege Üniversitesi, Mühendislik Fakültesi, Bilgisayar Mühendisliği Bölümü, İzmir, Türkiye (ORCID: 0000-0002-4872-5698), hasan.bulut@ege.edu.tr
}

(International Conference on Design, Research and Development (RDCONF) 2021 - 15-18 December 2021)

(DOI: $10.31590 /$ josat.1039890)

ATIF/REFERENCE: Tanışman, S., Karcıoğlu, A. A., Uğur, A. \& Bulut, H. (2021). Bitcoin Fiyatının LSTM Ağı ve ARIMA Zaman Serisi Modeli Kullanarak Tahmini ve Karşılaştırılması. Avrupa Bilim ve Teknoloji Dergisi, (32), 514-520.

\section{$\ddot{O} \mathbf{z}$}

Finansal varlıkların gelecekteki değerlerinin tahmini yatırımcılar için varlıklarını korumak adına önemlidir. 2008 yılında hayatımıza giren ve finansal varlıklar konusunda radikal bir değişiklik olan Bitcoin ise eski ve yeni yatırımcıların ilgisini çekmiş durumdadır. Ancak Bitcoin, doğası gereği diğer finansal varlıklara göre değerini belirleyen farklı parametreler içermektedir ve geleneksel tahmin yöntemleri Bitcoin gibi çok hareketli değerlere sahip finansal varlıkları tahmin etmekte güçlük çekmektedir. Bu çalışmada çok değişkenli LSTM sinir ağı ve klasik ARIMA zaman serisi modeli kullanılarak Bitcoin’in gelecek değerinin tahmini için modeller geliştirilmiştir. Uygulanan iki modelin tahmin doğruluğu performans değerlendirme metrikleri olan hata metrikleri kullanılarak karşılaştırılmıştır. Deneysel çalışmalar sonucu, LSTM sinir ağı modeli yakın ve uzak gelecek için düşük hata oranı ile tahmin performansı gerçekleştirirken ARIMA zaman serisi modeli yakın gelecek tahmini için düşük hata oranı ile tahmin performansı gerçekleştirmiştir.

Anahtar Kelimeler: Zaman Serisi Tahmini, Makine Öğrenmesi, Bitcoin.

\section{Forecasting of Bitcoin Price Using LSTM Neural Network and ARIMA Time Series Models and Comparision of Methods}

\begin{abstract}
It is important to forecast the future value of financial assets for investors to protect their assets. Bitcoin, which introduced to our lives in 2008 and is a radical change in financial assets, has been attracted the attention of both old and new investors. However, by its nature, Bitcoin contains different parameters that determine its value compared to other financial assets, and traditional forecasting methods have difficulty in predicting future values with very volatile financial assets such as Bitcoin. In this study, multivariate LSTM neural network and classic ARIMA time series model to forecast the future value of Bitcoin have been developed. The prediction accuracy of the two models applied has been compared using error metrics, which are performance evaluation metrics. As a result of the experimental studies, the LSTM neural network model has been performed prediction performance with a low error rate for the near and far future, while the ARIMA time series model has been performed prediction performance with a low error rate for the near future prediction.
\end{abstract}

Keywords: Time Series Forecasting, Machine Learning, Bitcoin.

* Sorumlu Yazar: sezercan.tanisman@ege.edu.tr 


\section{Giriş}

Bitcoin [1], ilk olarak 2008 yılında ortaya çıkmıştır ve günümüzde hızla hayatımızda yeni bir yatırım aracı olarak yer almıştır [2]. Bitcoin uçtan uca, merkezi otoritenin bulunmadığı bir dijital varlıktır. Bitcoin'deki tüm işlemler blokzinciri adı verilen merkeziyetsiz sistemde barınırlar. Bitcoin'in zamana bağlı değeri, tıpkı borsa gibi birçok farklı etkene bağlıdır [3]. Ancak borsada değeri genelde ticari etmen belirlerken, Bitcoin'de ise etmenler borsaya göre daha farklıdır. Ayrıca, borsadan farklı olarak, Bitcoin tarihi boyunca borsalarda göremeyeceğimiz yüksek sıçramalar ve düşüşler kaydetmiştir. Bu nedenler Bitcoin'in gelecek değerini tahmin etmekte zorluklar oluşturmaktadır.

Bu çalışmada finans alanından da yardım alarak Bitcoin'in gelecek değerlerinin tahmin edilmesi hedeflemiştir. Çalışmada LSTM sinir ağı ve ARIMA zaman serisi modelleri kullanılmıştır. Günlük aralıklarla oluşturulmuş veri seti üzerinde modeler uygulanmıştır. Geliştirilen LSTM ağ modelinin gerçekleştirimi için Tensorflow Keras [4] ortamı kullanılmıştır.

Çalışmanın devamında; 2. bölümde ilgili çalışmalardan bahsedilmiştir, 3. bölümde çalışmada kullanılan materyaller ve çalışmanın yöntemi ifade edilmiştir, 4. bölümde deneysel çalışmalar ve 5 . bölümde ise elde edilen sonuçlar paylaşılmıştır ve gelecek çalışmalardan bahsedilmiştir.

\section{2. İlgili Çalışmalar}

\subsection{Zaman Serisi Tahminleme}

Siami-Namin ve Namin yaptıkları çalışmada [5] zaman serisi tahminleme için ARIMA ve LSTM modellerini karşılaştırmışlardır. Bu karşılaştırma finans alanında zaman serisi verileri kullanılarak gerçekleştirilmiştir. Deneysel çalışmalar sonucu iki modelin de ortalama karekök sapması hesaplanmış ve karşılaştırılmıştır. LSTM modelinde, ARIMA modeline göre hata oranında $84 \%-87 \%$ arasında düşüş elde edilmiştir. Ayrıca çalışmada LSTM modelinin sonucu üzerinde epoch, batch boyutu vb. hiper parametrelerin etkisinden bahsedilmiştir.

Yunpeng ve arkadaşları [6] farklı veri kalıpları ile ARIMA, GRNN ve LSTM kullanarak çok adımlı zaman serilerinin tahminini gerçekleştirmişlerdir. Elde ettikleri sonuçlar 1şığında LSTM modelinin zaman tahminlemesinde geleneksel yöntemlere göre daha geniş veri kalıbı yelpazesine sahip, modelleme sürecinin hızlı ve tahminleme doğruluğunun daha yüksek olduğunu belirtmişlerdir. Öte yandan, geleneksel istatistiksel tabanlı yöntemlere göre daha çok kaynak gerektirdiğini ve tahminleme hızının daha yavaş olduğunu belirtmişlerdir.

\subsection{Bitcoin Fiyat Tahmini}

Valenkar ve arkadaşları [7] Bayesian regresyonu ve rastgele orman makine öğrenmesi algoritmalarını kullanarak Bitcoin'in gelecek değerlerini tahminlemek için bir model önermişlerdir. Önerdikleri modeli eğitmek için Bitcoin'in blok boyutu, toplam Bitcoin, günlük yüksek, işlem sayısı ve işlem hacmi özelliklerini kullanmışlardır. Eğitim verisi logaritmik, standart sapma, z-skoru ve box-cox normalizasyon yöntemleri ile normalize edilmiştir.

McNally ve arkadaşları [8] Bitcoin'in gelecek değerlerinin tahmini için RNN, LSTM ve ARIMA modellerini kullanmışlardır ve ortalama karekök sapması ile tahmin performanslarını karşılaştırmışlardır. En yüksek hata ARIMA ile elde edilirken, en yüksek hata ise RNN ile elde edilmiştir.

Azari yaptığı çalışmada [9] ARIMA kullanarak Bitcoin fiyat tahmini yapmıştır. Yazar çalışmada önce ARIMA (p, q, d) modeli için tahmin hatasını en aza indirecek p, q, d değerlerini bulmak için ızgara arama yöntemi kullanmıştır, daha sonra bu değerleri kullanarak tahmin hatasını en aza indirecek zaman penceresini bulmak için tekrar ızgara arama yöntemi kullanmıştır. Çalışmada, 1zgara aramada tahmin hatası hesaplamak için artık kareler toplamı ve ortalama kare hata yöntemleri ayrı ayrı kullanılarak 2 farklı model oluşturulmuştur ve bu iki modelin tahmin performansları karşılaştırılmıştır.

Chen ve arkadaşları [10] tarafından yapılan çalışmada Bitcoin gelecek değer tahmini için; lojistik regresyon, doğrusal ayırma analizi (LDA), rastgele orman, XGBoost (XGB), ikinci dereceden ayrım analizi, destek vektör makinesi (SVM) ve LSTM yöntemlerini karşılaştırmaktadır. Çalışmada modeller doğruluk, kesinlik, duyarlılık ve F1 skoru ile karşılaştırılmıştır. Modeller günlük veriler ile eğitildiğinde istatistiksel modeller olan LR ve LDA ortalama $66 \%$ doğruluk; makine öğrenmesi yöntemlerinden en iyi doğruluk 65.3\% ile SVM'den, en kötü doğruluk 48.3\% ile XGB'den elde edildiği belirtilmiştir. Diğer yandan modeller 5 dakika aralıklı veriler ile eğitildiğinde LSTM modelinin $67.2 \%$ ile en iyi doğruluğa sahip olduğu görülmüştür. Makine öğrenmesi algoritmaları ortalama $62.2 \%$ doğruluk elde ederken, istatistiksel yöntemler ortalama doğruluğu 53\% olarak elde etmiştir.

\section{Materyal ve Yöntemler}

\subsection{Veri Seti}

Çalışmada kullanılan veri seti 29 Nisan 2013 tarihinden başlayarak 27 Şubat 2021 tarihine kadar, toplamda 2862 günlük, Bitcoin'in açılış, en yüksek, en düşük, kapanış, hacim ve market değeri özelliklerini içermektedir. $\mathrm{Bu}$ veri seti Kaggle [11] platformundan elde edilmiştir. Eğitim verisi hazırlamak için veri setine finans alanında kullanılan teknik analiz değerleri de eklenmiştir. Bu eklenen değerlerden birisi olan bağıl güç endeksi (relative strength index/RSI) belirli bir zaman periyodu içinde bir varlığın aşırı alınmışlığını veya aşırı satılmışlığını ifade eden istatistiksel bir değerdir. RSI, seçilen zaman periyodu içindeki varlığın yukarıya doğru ve aşağıya doğru hareketlerinin ayrı ayrı ortalamalarının birbirine bölümü ile hesaplanır ve bu değerle ters orantılıdır. Diğer bir kullanılan teknik analiz değeri ise üstel hareketli ortalama (exponential moving average/EMA) değeridir. Belirli bir zaman periyodu içinde varlığın EMA değeri Denklem 1-2 ile gösterilen formül ile hesaplanır.

$k=\frac{\text { Smoothing }}{\text { (periyot }+1)}$ olmak üzere

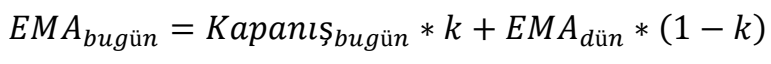

RSI ve EMA için periyot olarak 14 gün seçilerek hesaplanmış ve eğitim verisi hazırlamak için veri setine eklenmiştir. İlk 14 gün için RSI hesaplanamayacağı için eğitim verisinden çıkarılmıştır.

14 gün periyot seçimi Wilder'in yaptığı çalışmada [12] önerdiği üzere seçilmiştir. Veri setinin son hali Şekil 1'de gösterilmiştir. Veri seti \%80 eğitim verisi, \%20 test verisi olarak ayrılmıştır. Eğitim verisi min-max normalizasyonu ile $[0,1]$ aralığında normalize edilmiştir. 


\begin{tabular}{|c|c|c|c|c|c|c|c|}
\hline & rih & Açılış & En Düşük & En Yüksek & Kapanış & RSI-14 & EMA-14 \\
\hline 0 & $12-05-1323: 59$ & 115.639999 & 113.434998 & 117.448998 & 115.0 & 33.283783 & 118.515 \\
\hline 1 & $13-05-1323: 59$ & 114.82 & 114.5 & 118.698998 & 117.980003 & 34.345796 & 117.615664 \\
\hline 2 & $14-05-1323: 59$ & 117.980003 & 110.25 & 119.800003 & 111.5 & 35.598777 & 118.845798 \\
\hline 3 & $15-05-1323: 59$ & 111.400002 & 103.5 & 115.809998 & 114.220001 & 45.267357 & 119.268002 \\
\hline 4 & $16-05-1323: 59$ & 114.220001 & 112.199997 & 118.760002 & 118.760002 & 63.999216 & 116.203332 \\
\hline$\ldots$ & & $\ldots$ & $\ldots$ & $\ldots$ & $\ldots$ & $\ldots$ & $\ldots$ \\
\hline 2844 & 23-02-21 23:59 & 54204.92976 & 45290.59027 & 54204.92976 & 48824.42687 & 59.884414 & 57089.594721 \\
\hline 2845 & $24-02-2123 \cdot 59$ & 48835.08766 & 47213.49816 & 51290.13669 & 49705.33332 & 58.582395 & 53816.290684 \\
\hline 2846 & $25-02-2123: 59$ & 49709.08242 & 47093.85302 & 51948.96698 & 47093.85302 & 49.205685 & 51377.980729 \\
\hline 2847 & $26-02-2123-59$ & 47180.46405 & 44454.84211 & 48370.78526 & 46339.76008 & 50.446376 & 51471.876084 \\
\hline 2848 & $27-02-21 \quad 23: 59$ & 46344.77224 & 45269.02577 & 48253.2701 & 46188.45128 & 47.14023 & 48355.116572 \\
\hline
\end{tabular}

Şekil 1. Bitcoin günlük değerleri veri seti

\subsection{LSTM ile Bitcoin Fiyat Tahmini}

LSTM ağları, RNN ağları ile çok benzer olan ancak RNN ağlarının karşılaştığı sorunları çözmek için geliştirilmiş yapay sinir ağıdır. RNN ağları bir önceki adımın çıktısını mevcut adımın girdisi olarak kullanırlar ve kısa bir süre belleğinde tutarlar. $\mathrm{Bu}$ sayede doğal dil işleme ve zaman serileri kapsamındaki problemlerin çözülmesinde kullanılabilirler. Ancak RNN ağları bilgiyi uzun süreli tutmakta başarısız olurlar. Bu nedenle önemli bazı bilgiler ağın başında kaybolabilir. Aynı zamanda RNN ağları, geri yayılım süresince gradyan yokolması problemi ile de karşılaşmaktadırlar. LSTM ağları bahsedilen bu problemlerin üstesinden gelmektedirler. LSTM hücreleri kısa süreli bellek sorununu çözmek için bilgi akışını düzenleyen ve kontrol eden kapılar oluştururlar. Aşağıda görevleri belirtilen [13] bir hücre girdi kapısı, hücre durumu, unutma kapısı ve çıktı kapısı içerir ve bu kapılar sigmoid veya tanh aktivasyon fonksiyonu kullanır.

1. Girdi kapısl: Hücre durumunu güncellemek için bir önceki gizli durumu ve şu anki girdiyi alarak sigmoid fonksiyonundan geçirir.

2. Hüсre durumu: Diğer kapıların yardımıyla bilgiyi tutmayı veya unutmayı sağlar.

3. Çıktı kapısı: Hücrenin çıktısını oluşturur.

4. Unutma kapısı: Bilginin unutulacak ve tutulacak parçalarına karar verir.

Girdi kapısı, unutma kapısı, çıktı kapısı hücre durumu ve gizli durum değerlerini sırasıyla $i_{t}, f_{t}, o_{t}, c_{t}$ ve $h_{t}$ gösteriyor olsun. $x_{t} \mathrm{t}$ zamanındaki girdi vektörü, $\sigma$ sigmoid aktivasyon fonksiyonu ve $\mathrm{W}, \mathrm{b}$ parametre matrisi ve vektörü olmak üzere Şekil 2'de görülen LSTM hücresi Denklem 3-7 kullanılarak tanımlanabilir. $[14,15]$

$i_{t}=\sigma\left(W_{x i} x_{t}+W_{h i} h_{t-1}+W_{c i} c_{t-1}+b_{i}\right)$

$f_{t}=\sigma\left(W_{x f} x_{t}+W_{h f} h_{t-1}+W_{c f} c_{t-1}+b_{f}\right)$

$c_{t}=f_{t} \odot c_{t-1}+i_{t} \odot \tanh \left(W_{x c} x_{t}+W_{h c} h_{t-1}+b_{c}\right)$

$o_{t}=\sigma\left(W_{x o} x_{t}+W_{h o} h_{t-1}+W_{c o} c_{t}+b_{o}\right)$

$h_{t}=o_{t} \odot \tanh \left(c_{t}\right)$

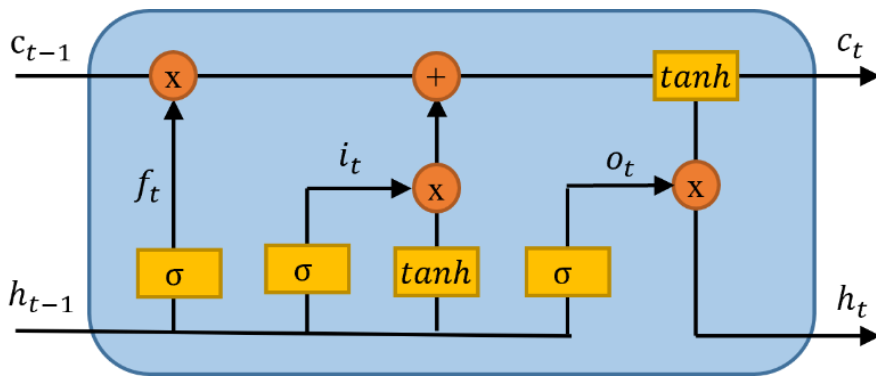

Şekil 2. Bir LSTM Hücresi

Çalışmamızda LSTM sinir ağı kullanarak tahminleme gerçekleştirmek için çok değişkenli zaman serisi modeli geliştirilmiştir. Şekil 3'te gösterildiği gibi, geliştirlen model, tanh aktivasyonuna sahip 3 LSTM ağ katmanına ve her bir katman için aşırı öğrenmeyi engellemek için \%20 dropout'a sahiptir. Model Tensorflow Keras ortamında gerçekleştirilmiştir. Her bir LSTM katmanındaki hücre sayısı Keras hiperparametre optimizasyonu kullanılarak elde edilmiştir. Modelde Adam optimizasyonu kullanılmıştır ve öğrenme katsayısı $\beta=0.001$ seçilmiştir.

Eğitime katılan teknik analiz değerlerinin tahmin performansındaki etkisini ölçmek amacıyla model ilk olarak açılış, en düşük, en yüksek, kapanış ve RSI-14 değerleri kullanılarak, ardından ise bu değerlere EMA-14 değeri de eklenerek eğitilmiştir.

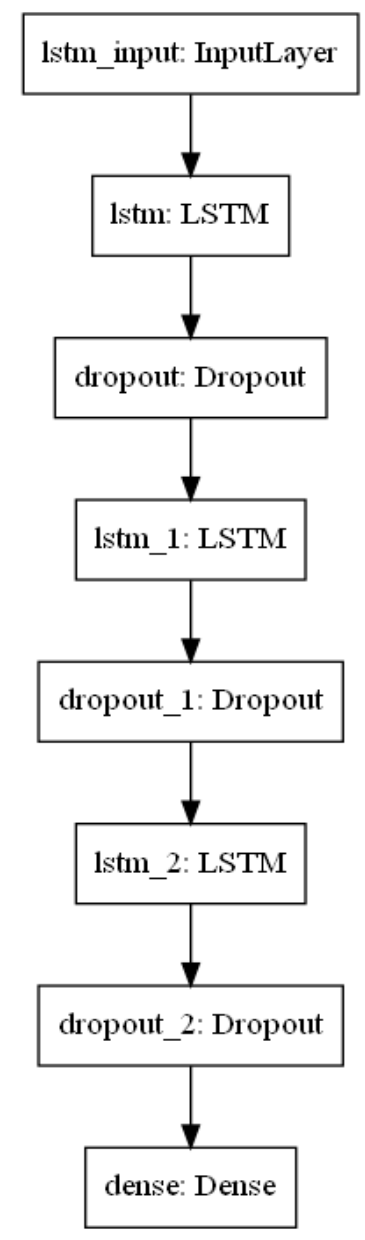

Şekil 3. Önerilen LSTM sinir ă̆ı modeli 


\subsection{ARIMA ile Bitcoin Fiyat Tahmini}

Box Jenkins tarafından önerilen Box-Jenkins tekniği, zaman serilerinin analizi için yaygın olarak kullanılmaktadır [16]. Bu teknik, durağan olmayan modeller olan serilere uygulanan otoregresif hareketli ortalama (ARIMA) modellerini içerir, ancak modeller, seri fark işlemi ile durağan hale getirilir. Box-Jenkins yaklaşımının temeli, ele alınan verilerin doğasına bağlı olarak, çeşitli model seçenekleri arasından en uygun parametreyi içeren ancak aynı zamanda sınırlı parametreyi içeren bir ARIMA modeli seçmektir.

$\operatorname{ARIMA}(\mathrm{p}, \mathrm{d}, \mathrm{q})$ modelleri, d derecesinden seri farkı alınarak ve ARMA'nın stabilizasyon süreci modeli $(p, q)$ eklenerek elde edilir. ARIMA (p, d, q) modellerinde, $p$, otoregresif (AR) model derecesidir, q, hareketli ortalama (MA) model derecesidir ve d, seriyi durağan hale getirmek için gereken fark sayısıdır. Zaman serisi durağan ise, ARIMA modeli AR (p), MA(q) veya ARMA $(\mathrm{p}, \mathrm{q})$ olur.

ARIMA modelinin $(1,0,0)$ modeli bu çalışmada kullanılmıştır ve veri setinin son \%20'lik kısmı tahmin edilecek gün sayıs1 olarak belirlenmiştir. Veri setinin geri kalan kısmının tamamı eğitim için kullanılmıştır. ARIMA modelinde gelecekteki doğrulanmış Bitcoin fiyatlarını tahmin etme modeli Denklem 8'de tanımlanmıştır.

$\operatorname{ARIMA}(\mathrm{p}, \mathrm{d}, \mathrm{q}): X_{t}=\alpha_{1} X_{t-1}+\alpha_{2} X_{t-2}+\beta_{1} Z_{t-1}+\beta_{2} Z_{t-2}+$ $Z_{t}$ ve $Z_{t}=X_{t}-X_{t-1}$

Burada $X_{t}, t$. günde tahmini doğrulanmış Bitcoin fiyatı, $\alpha_{1}$, $\alpha_{2}, \beta_{1}$ ve $\beta_{1}$ parametrelerdir, $Z_{t}$ ise $t$. gün için kalan terimdir.

Çalışmada kullanılan ARIMA zaman serisi modelinin genel işleyişi Şekil 4'te gösterilmiştir.

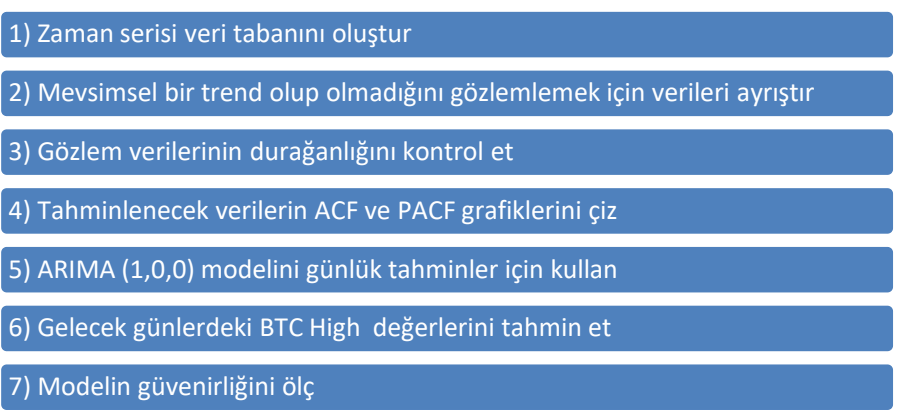

\section{Deneysel Çalışmalar}

Deneysel çalışmaları gerçekleştirmek için Python programlama dilinin 3.8 .8 versiyonu kullanılmıştır. Tüm deneyler AMD Ryzen 7 2700X @ 3.7GHz CPU, 16 GB RAM ve NVDIA GeForce GTX 1060 3GB GPU donanımlarına sahip bir makinede gerçekleştirilmiştir.

\subsection{LSTM ile Tahmin Deneyleri}

Geliştirilen model 14 günlük zaman adımları ile ilk olarak günlük açılış, en yüksek, en düşük, kapanış girdi değerleri kullanılarak 14x4 matrisler eğitilmiştir ve Şekil 5'te gösterilmiştir. Ardından teknik analiz verilerinin kullanılmasının tahmin performansına etkisinin ölçülmesi için RSI-14 ve EMA14 değerleri ayrı ayrı eklenerek 14x5 matrisler ile model yeniden eğitilmiştir, sırasıyla Şekil 6 ve Şekil 7'de gösterilmiştir. Son olarak da Şekil 8'de görüldüğü üzere RSI-14 ve EMA-14 beraber kullanılarak 14x6 matrisler ile model yeniden eğitilmiştir.

Tüm eğitimler sonunda modelin tahmin performansını ölçmek için Denklem 9'da formülü verilmiş olan ortalama mutlak yüzde hata (mean absolute percentage error/MAPE), Denklem 10 'da formülü verilmiş olan ortalama mutlak hata (mean absolute error/MAE) ve Deklem 11'de formülü verilmiş olan ortalama karekök hatası (root mean square error/RMSE) kullanılmıştır. Elde edilen sonuçlar Tablo 1'de verilmiştir. Denklem 9, 10 ve 11 için $\mathrm{G}$ gerçek değeri ve $\mathrm{T}$ modelin tahmin ettiği değeri ifade etmektedir.

$$
\begin{aligned}
& M A P E=\frac{1}{n} \sum_{t=1}^{n}\left|\frac{T_{t}-G_{t}}{G_{t}}\right| \\
& M A E=\frac{1}{n} \sum_{t=1}^{n}\left|T_{t}-G_{t}\right| \\
& R M S E=\sqrt{\frac{\sum_{t=1}^{n}\left(T_{t}-G_{t}\right)^{2}}{n}}
\end{aligned}
$$

Şekil 4. ARIMA Modeli Genel Işsleyişi

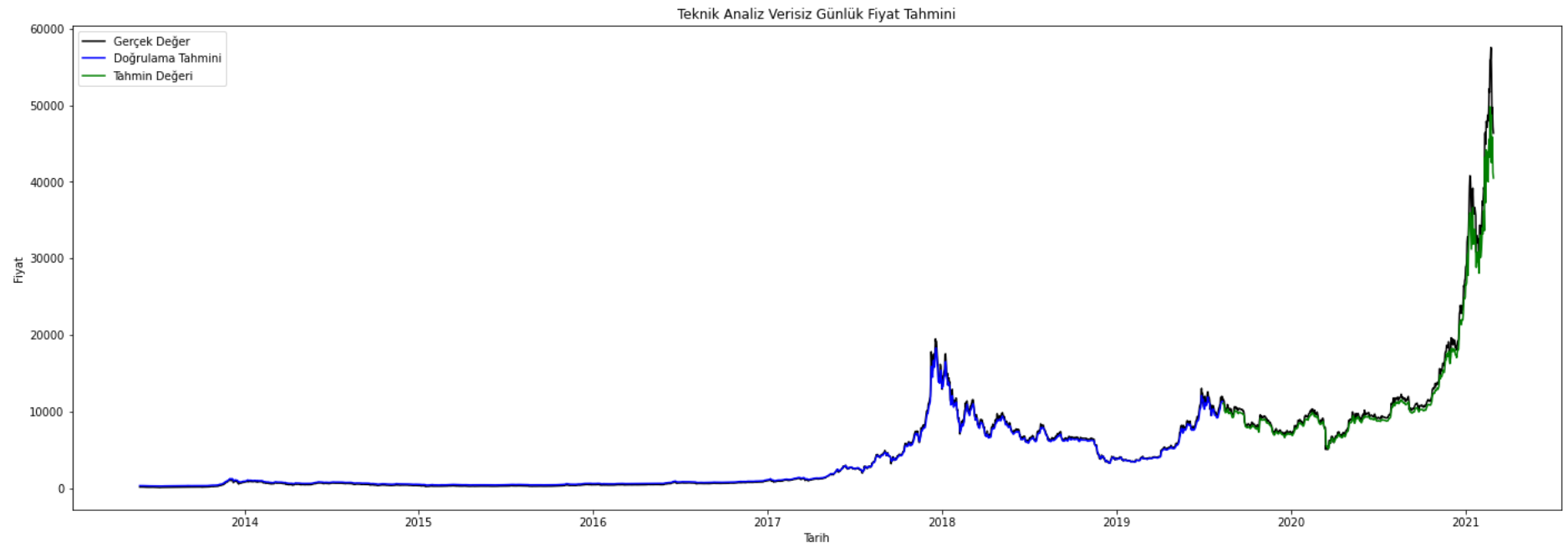


Şekil 5. Teknik analiz verisi kullanmadan eğitilen modelde fiyat tahmini.

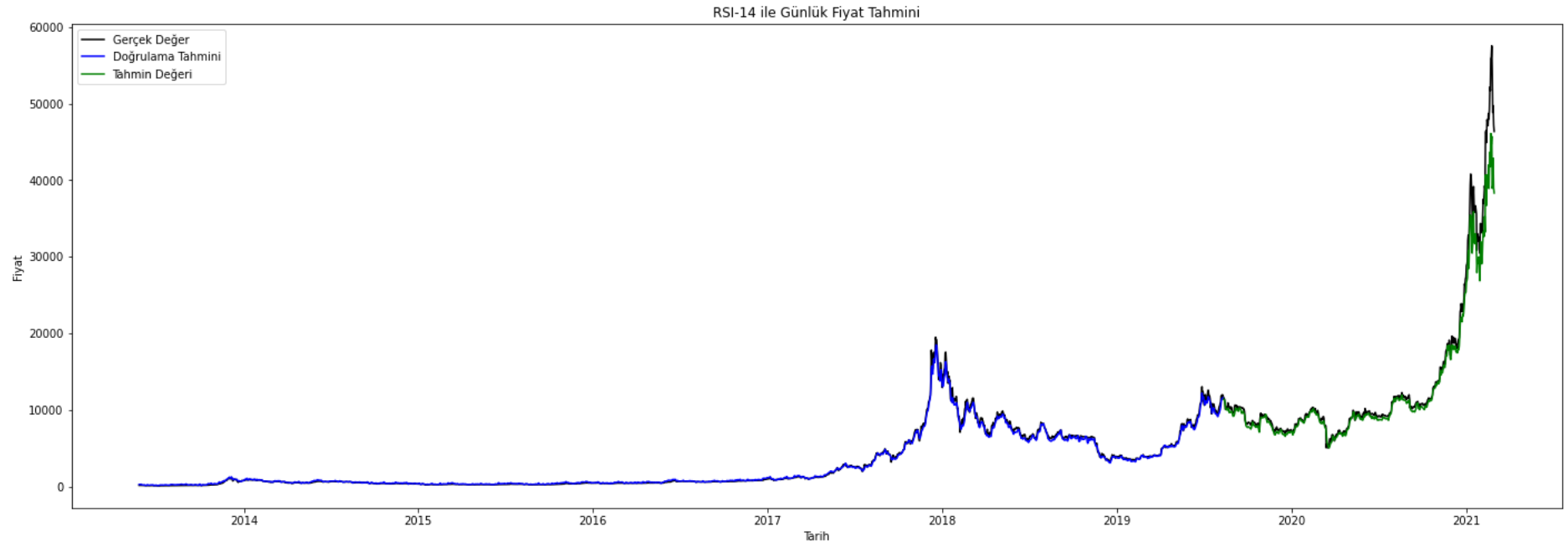

Şekil 6. RSI-14 kullanilarak eğitilen modelde fiyat tahmini

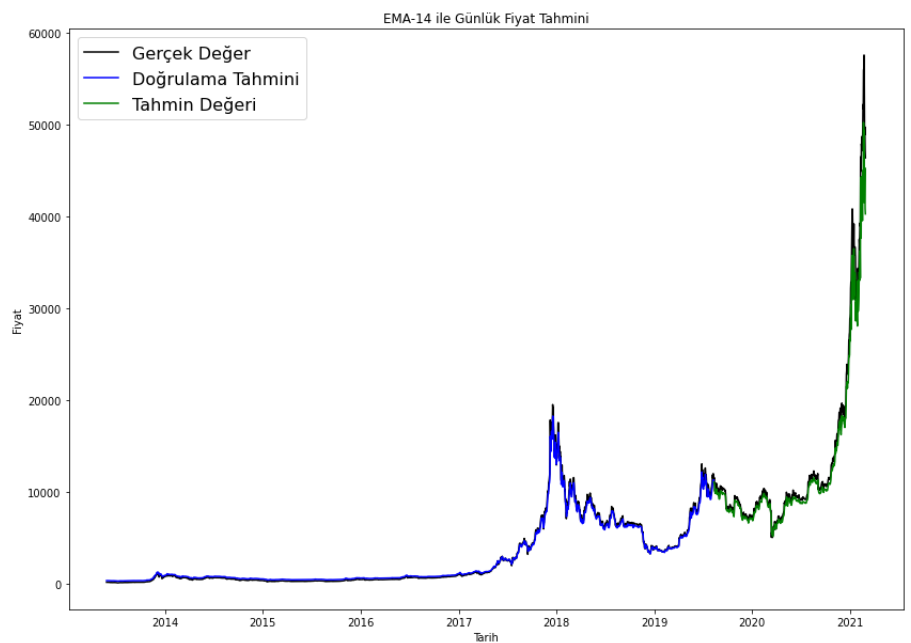

Şekil 7. EMA-14 kullanılarak eğitilen modelde fiyat tahmini

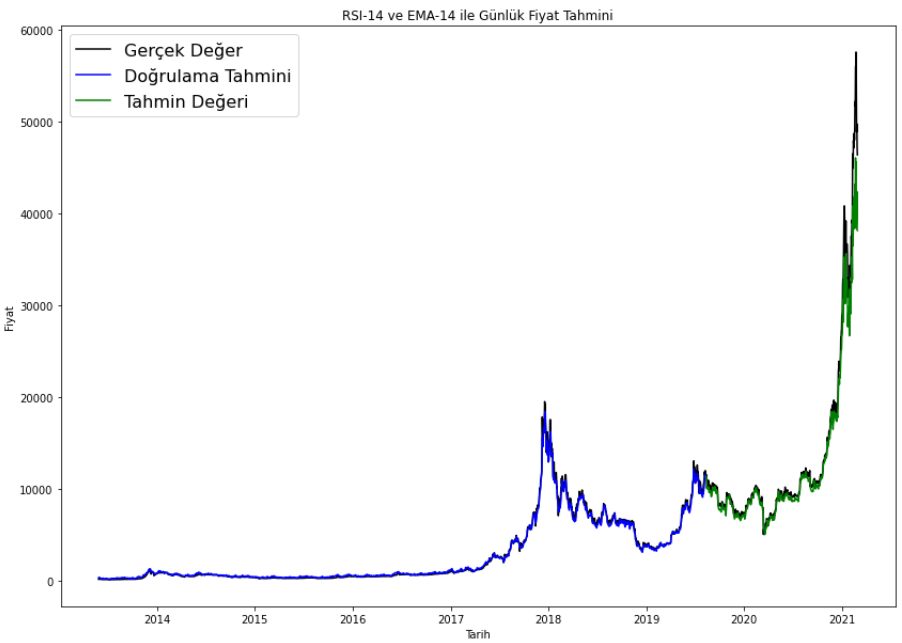

Şekil 8. RSI-14 ve EMA-14 kullanılarak eğitilen modelde fiyat tahmini

Tablo 1. Deneyler çalışmalar sonucunda tüm modellerden elde edilen ölçek değerleri

\begin{tabular}{|c|c|c|c|c|c|c|c|}
\hline \multicolumn{2}{|c|}{ Eğitim Verisi Özellikleri } & \multicolumn{2}{|c|}{ Doğrulama Tahmini Ölçekleri } & \multicolumn{3}{c|}{ Test Tahmini Ölçekleri } \\
\hline RSI-14 & EMA-14 & MAPE & MAE & RMSE & MAPE & MAE & RMSE \\
\hline Mevcut Değil & Mevcut Değil & $34.30 \%$ & 128.61 & 348.45 & $4.96 \%$ & 865.48 & 1613.93 \\
\hline Mevcut & Mevcut Değil & $17.02 \%$ & 172.73 & 333.88 & $4.76 \%$ & 912.57 & 1994.26 \\
\hline Mevcut Değil & Mevcut & $35.10 \%$ & 224.52 & 354.23 & $5.14 \%$ & 896.95 & 1686.64 \\
\hline Mevcut & Mevcut & $23.28 \%$ & 195.56 & 348.23 & $5.12 \%$ & 970.8 & 2079.2 \\
\hline
\end{tabular}

\subsection{ARIMA ile Tahmin Deneyleri}

Çalışmada zaman serisi veri tabanı oluşturulduktan sonra mevsimsel bir trend olup olmadığını gözlemlemek için verilerin ayrıştırma grafikleri çizilmiştir. BTC High değerinin trend eğilimi Şekil 9'da gösterildiği gibidir. 2017 senesi sonunda yükselişe geçen ve 2018 senesi sonu tekrar eski değerlerine yakın bir değere doğru düşüşe geçtiği gözlemlenmektedir.

ARIMA modelinin günlük fiyat verileri üzerinde normallik ve durağanlıktaki varyansı için test edilmiştir. Tahmin yapmadan önce gözlem verilerinin durağanlı̆g kontrol edilmiştir. Ayrıca günlük tahmini yapılan her bir verinin durağanlığını sağlamak 
için yapılan istatistiksel bir test olan Dickey-Fuller testleri yapılmıştır. Şekil 10 günlük fiyat değerlerinin durağanlığını göstermektedir. Yapılan analiz sonu fiyat değerlerinin durağanlık göstermediği gözlemlenmiştir.
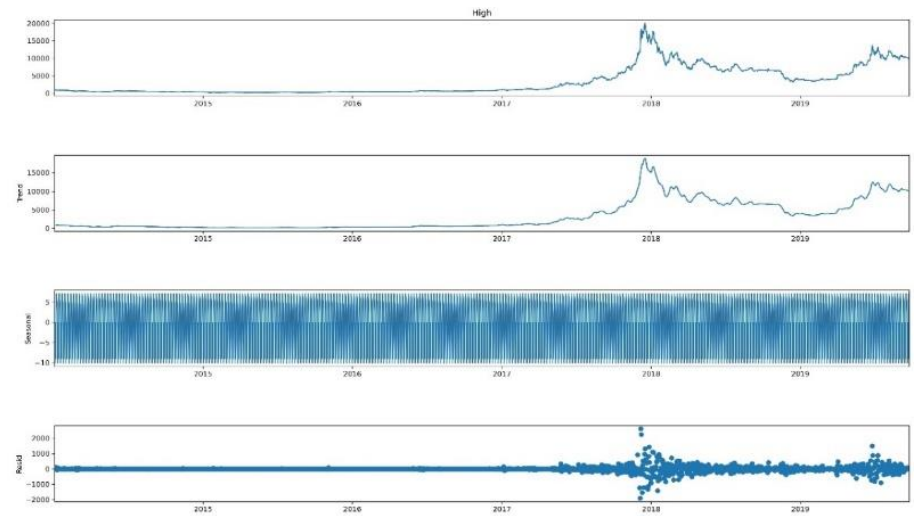

Şekil 9. Günlük fiyatın mevsimsel trend grafiği

Results of Dickey-Fuller Test:

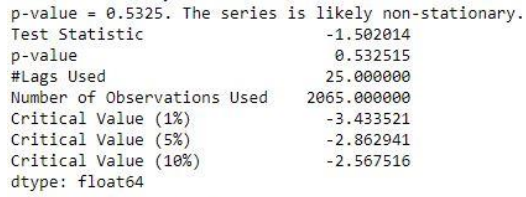

<Figure size $864 \times 288$ with $\theta$ Axes>

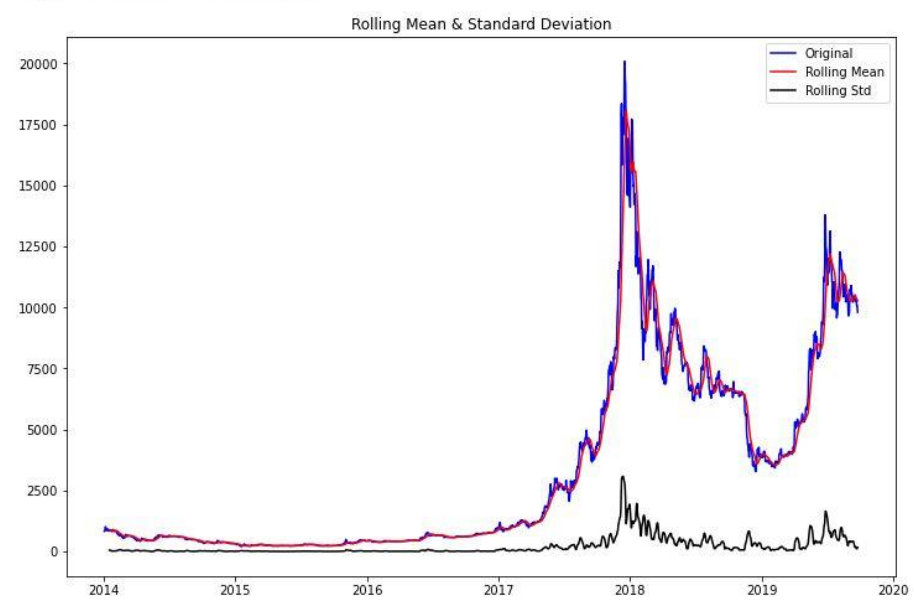

Şekil 10. Günlük fiyat değerinin durăganlık ve DF testi

ARIMA modellerinin başlangıç sayısını bulmak için otokorelasyon fonksiyonu (ACF) grafiği ve kısmi otokorelasyon (PACF) grafiği Şekil 11'de gösterildiği gibi elde edilmiştir. Otokorelasyon fonksiyonu, olası tüm gecikmelerde otokorelasyonu verir. 0 gecikmesindeki otokorelasyon, varsayılan olarak dahil edilir ve bu, her zaman veriler ve kendileri arasındaki korelasyonu temsil eden 1 değerini alır.

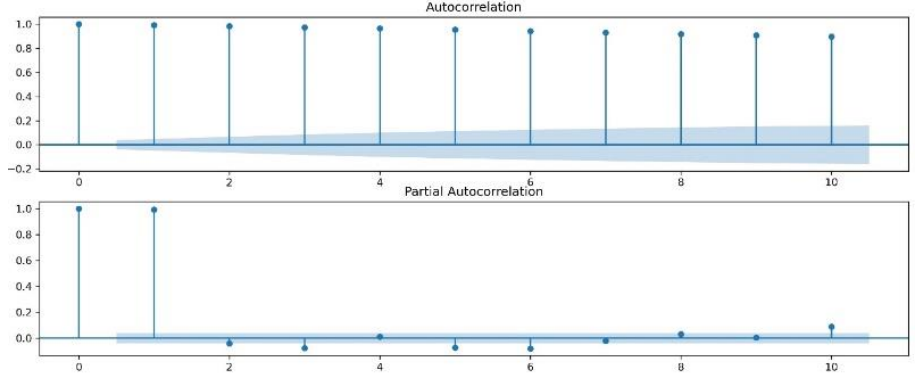

Şekil 11. Günlük fiyat değerinin oto \& klsmi korelasyon grafiği e-ISSN: 2148-2683
ARIMA zaman serisi modelini kullanarak günlük fiyat değerinin tahmini azalmaya yönelik bir regresyon eğrisi Şekil 12 'de gösterildiği gibi elde edilmiştir.

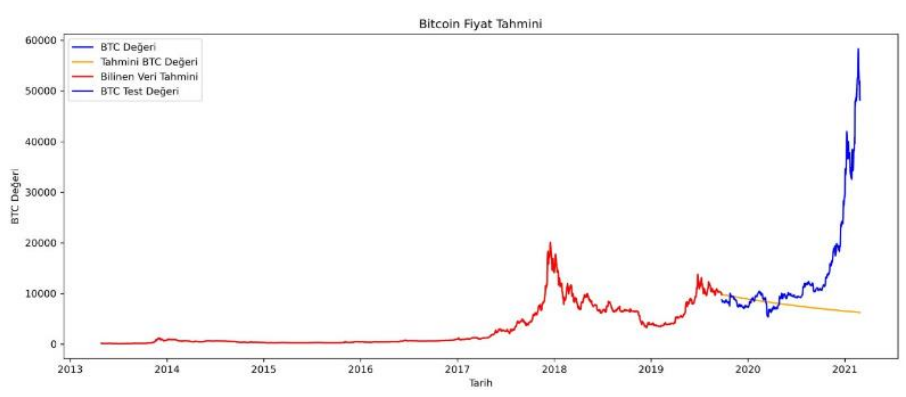

Şekil 12. ARIMA modeli ile günlük fiyat tahmini

Modelin tahmin doğruluğunu göstermek için performans değerlendirme metriklerinden Denklem 9'da formülü verilmiş olan MAPE ölçeği kullanılmıştır. Günlük veri setini kullanılarak eğitilen ARIMA modelinin test verisi kullanılarak tahmin performansı ölçüldüğünde MAPE ölçeğinde $33.47 \%$ hata değeri elde edilmiştir.

\section{Sonuçlar ve Gelecek Çalışmalar}

Olağanüstü durumlar olmadığı sürece bir finansal varlığın değeri ortalama olarak tahmin edilebilir. Ancak Bitcoin gibi günlük hayatımıza yavaş yavaş entegre olan kripto paraların değerleri çok hareketli olabilmekteler. Bu çalışmada Bitcoin'in gelecekteki değerinin tahmin edilmesi için LSTM sinir ağı ve ARIMA zaman seri modeli olmak üzere iki farklı model uygulanmıştır.

Çalışmada LSTM modeli çok değişkenli zaman serisi yöntemi kullanılarak geliştirilmiştir ve farklı değişkenlerin kombinasyonun tahmin performansına etkisini ölçmek hedeflenmiştir. Gerçekleştirilen deneysel çalışmalar sonucu, LSTM sinir ağı modelinde teknik analiz verisi olarak sadece RSI14 kullanıldığında 4.76\% MAPE ve 912.57 MAE ile en iyi tahmin performansı elde edilmiştir, ancak RMSE RSI-14 kullanılmadığında daha düşük elde edilmiştir. RSI-14 kullanıldığında keskin iniş ve çıkışların yüksek hatayla tahmin edildiği gözlemlenmiştir. EMA-14 kullanıldığında genel olarak EMA-14 kullanımının tahmin performansına pozitif bir katkısı elde edilmemiştir. Öte yandan, hiçbir teknik analiz verisi kullanılmayan deneyi incelediğimizde, eğitilmiş modelin kötü olmayan bir tahmin performansı elde edilmiştir.

ARIMA zaman serisi modeli ile tahmin gerçekleştirildiğinde elde edilen sonucun yakın gelecek zaman için gerçek değerler ile aynı yönde olduğunu gözlemlenmiştir. Ancak uzak gelecek tahmininde ARIMA modeli yüksek hatalı tahminler gerçekleştirmiştir. ARIMA modeli tek değişkenli zaman serisi yöntemi olduğundan dolayı Bitcoin gibi çok hareketli ve birden fazla değişkene bağlı değeri olan varlıkların tahminlenmesi ARIMA modelin için uygun olmadığını söyleyebiliriz.

Gelecekte yapılacak çalışmalarda, tahmin performansının geliştirilmesi adına mevcut kullanılan teknik analiz değerleri farklı zaman periyotları ile verdiği sonuçlar incelenebilir, farklı teknik analiz değerleri ve kombinasyonları incelenebilir. ARIMA yerine ARIMAX modeli kullanılarak çok değişkenli zaman serisi tahmini yapılabilir. Modeller günlük veriler yerine saatlik, dakikalık vb. gibi daha sık veriler ile eğitilebilir. 


\section{Kaynakça}

[1] Nakamoto, S. (2008). Bitcoin: A peer-to-peer electronic cash system. Decentralized Business Review, 21260.

[2] Hong, K. (2017). Bitcoin as an alternative investment vehicle. Information Technology and Management, 18(4), 265-275.

[3] Huang, J. Z., Huang, W., \& Ni, J. (2019). Predicting Bitcoin returns using high-dimensional technical indicators. The Journal of Finance and Data Science, 5(3), 140-155.

[4] Chollet, F., \& others. (2015). Keras. GitHub. Retrieved from https://github.com/fchollet/keras

[5] Siami-Namini, S., \& Namin, A. S. (2018). Forecasting economics and financial time series: ARIMA vs. LSTM. arXiv preprint arXiv:1803.06386.

[6] Yunpeng, L., Di, H., Junpeng, B., \& Yong, Q. (2017, November). Multi-step ahead time series forecasting for different data patterns based on LSTM recurrent neural network. In 2017 14th web information systems and applications conference (WISA) (pp. 305-310). IEEE.

[7] Velankar, S., Valecha, S., \& Maji, S. (2018, February). Bitcoin price prediction using machine learning. In 2018 20th International Conference on Advanced Communication Technology (ICACT) (pp. 144-147). IEEE.

[8] McNally, S., Roche, J., \& Caton, S. (2018, March). Predicting the price of bitcoin using machine learning. In 2018 26th euromicro international conference on parallel, distributed and network-based processing (PDP) (pp. 339-343). IEEE.

[9] Azari, A. (2019). Bitcoin price prediction: An ARIMA approach. arXiv preprint arXiv:1904.05315.

[10] Chen, Z., Li, C., \& Sun, W. (2020). Bitcoin price prediction using machine learning: An approach to sample dimension engineering. Journal of Computational and Applied Mathematics, 365, 112395.

[11] Kaggle, https://www.kaggle.com/

[12] Wilder, J. W. (1978). New concepts in technical trading systems. Trend Research.

[13] Selvin, S., Vinayakumar, R., Gopalakrishnan, E. A., Menon, V. K., \& Soman, K. P. (2017, September). Stock price prediction using LSTM, RNN and CNN-sliding window model. In 2017 international conference on advances in computing, communications and informatics (icacci) (pp. 1643-1647). IEEE.

[14] Graves, A. (2013). Generating sequences with recurrent neural networks. arXiv preprint arXiv:1308.0850.

[15] Karevan, Z., \& Suykens, J. A. (2020). Transductive LSTM for time-series prediction: An application to weather forecasting. Neural Networks, 125, 1-9.

[16] Box, G. E., Jenkins, G. M., Reinsel, G. C., \& Ljung, G. M. (2015). Time series analysis: forecasting and control. John Wiley \& Sons. 